\title{
Ethyl Acetate Extract from Tissue-Cultured Mountain Ginseng Adventitious Roots Inhibits In Vitro Platelet Aggregation in Whole Human Blood and Augments Peripheral Blood Flow in Mice
}

\author{
In Sun Lee ${ }^{1}$, Seul-Ki Kim ${ }^{1}$, Min Hwa Jeon ${ }^{2}$, and Won Kyung Jeon ${ }^{{ }^{*}}$ \\ ${ }^{1}$ Creative Research Laboratory, Korea Institute of Oriental Medicine, Daejeon 305-811, Korea \\ ${ }^{2} \mathrm{CBN}$ Biotech Co. Ltd., Cheongwon 363-883, Korea
}

\begin{abstract}
We previously reported that in vitro anti-platelet activity of tissue-cultured mountain ginseng (TCMG) ethanol extracts show improved efficacy when compared with commercial ginseng products such as Korean red ginseng and Panax ginseng. However, information on the anti-platelet activity of the ethyl acetate fraction from TCMG adventitious roots is limited. Therefore, in this study, we further investigated the effects of an ethyl acetate extract of TCMG (EA-TCMG) adventitious roots on in vitro antiplatelet activity in whole human blood and its effect on peripheral blood flow in mice. We found that EA-TCMG inhibited platelet aggregation with $\mathrm{IC}_{50}$ values of 271,180 , and $147 \mu \mathrm{g} / \mathrm{mL}$ induced by collagen, adenosine-5' -diphosphate, and arachidonic acid, respectively. Among the three agonists used, thromboxane $\mathrm{A}_{2}$ formation induced by arachidonic acid was markedly suppressed. Furthermore, EA-TCMG improved the peripheral circulatory disturbance by improving vascular blood flow. In conclusion, these results suggest that ethyl acetate extracts from TCMG adventitious roots might inhibit vascular platelet aggregation and thrombus formation.
\end{abstract}

Keywords: Panax ginseng, Platelet aggregation, Human blood, Tissue-cultured mountain ginseng

\section{INTRODUCTION}

Certain herbal medicines and natural products show promise as agents for improving blood viscosity and microcirculation, as well as preventing atherosclerotic diseases, such as myocardial and cerebral infarction [1]. Many studies have reported that modulation of platelet activity using specific pharmacological agents is a successful strategy for preventing thrombotic disorders [2]. Ginseng radix, the root of Panax ginseng Meyer, is one of the world's best known medicinal plants and has long been studied for its medical efficacy [3]. In Korea, mountain ginseng has traditionally been considered more effective than cultivated ginseng products [4]. Although mountain ginseng is well known in pharmaceutics, much

cc This is an Open Access article distributed under the terms of the Creative Commons Attribution Non-Commercial License (http://creativecommons.org/licenses/by-nc/3.0/) which permits unrestricted non-commercial use, distribution, and reproduction in any medium, provided the original work is properly cited. remains to be understood to establish a scientific foundation for traditional use $[5,6]$. Mountain ginseng has become extremely scarce; thus, the commercial ginseng supply depends almost exclusively on field cultivation, which is a time-consuming and labor-intensive process. However, tissue culture techniques for mountain $P$. ginseng have made bioreactor technology a useful tool for large-scale production of root biomass [6,7]. Tissue culture techniques have allowed us to easily obtain a mass of adventitious roots, which can then be studied for the various biological effects of tissue-cultured mountain ginseng (TCMG). Recent findings have demonstrated that TCMG aids in the prevention or treatment of sper-

Received 20 Jun. 2011, Revised 15 Sep. 2011, Accepted 15 Sep. 2011

\footnotetext{
*Corresponding author

E-mail: wkjeon@kiom.re.kr

Tel: +82-42-868-9505, Fax: +82-42-868-9573
} 
matogenic disorders [8,9], erectile dysfunction [10], symptoms of hyperlipidemia [11], and has anti-fibrotic and antioxidant activity $[12,13]$. In addition, TCMG stimulates immune cells and inhibits growth of cancer cells [14]. We recently reported that TCMG has more effective anti-platelet activity in $70 \%$ ethanol extracts than that of ginseng commercial products [15]. Platelets have multiple activation signaling mechanisms, so agents with anti-platelet activity may have wide therapeutic potential for blood flow disorders [16-18]. In this study, we found that ethyl acetate extract of TCMG (EA-TCMG) was a candidate for further study through confirmation of its in vitro effects on anti-platelet activity using whole human blood and in vivo peripheral blood circulation in a mouse model. Based on the results, we suggest that EATCMG shows higher anti-platelet potency than that of other TCMG solvent fractions, and that it also improves peripheral blood circulation.

\section{MATERIALS AND METHODS}

\section{Reagents and materials}

Catechin was purchased from Wako Pure Chemical Co. (Osaka, Japan). Collagen, adenosine-5'-diphosphate (ADP) and arachidonic acid (AA) were purchased from (Chrono-Log Co., Havertown, PA, USA). A thromboxane $\mathrm{B}_{2}\left(\mathrm{TXB}_{2}\right)$ ELISA kit was obtained from (R\&D Systems Inc., Minneapolis, MN, USA). The isofluran/foran solution (Choongwae Pharmaceutical Co., Seoul, Korea) was prepared by mixing oxygen (Shinheung Oxygen, Daejeon, Korea) and nitrogen (Shinheung Oxygen). TCMG was supplied by CBN Biotech in Ochang, Korea in 2008 and deposited at the TKM Converging Research Division, Korea Institute of Oriental Medicine. All other chemicals were purchased from Sigma-Aldrich (St. Louis, MO, USA).

\section{Extraction}

Dried roots of TCMG $(400 \mathrm{~g})$ were extracted with $70 \%$ ethanol and then concentrated under vacuum using a rotary evaporator. All extracts were freeze-dried to yield extract powders (121.1 g; yield, 30\%). The residue from the $70 \%$ ethanol extract was then extracted with nhexane, followed by trichloromethane ethyl acetate, nbutanol, and $\mathrm{H}_{2} \mathrm{O}$. Their yields were $3.38,2.78,2.35$, 28.69 , and $62.82 \%$, respectively.

\section{Volunteers}

Seven ( 1 female and 6 males; age range, 20 to 38 years) healthy volunteers entered the study. None of the subjects had a history of bleeding disorders or cardiovascular disease and refrained from any pharmacological therapy for at least $2 \mathrm{wk}$ prior to enrollment. None of the subjects smoked, nor did they exhibit hypertension, diabetes, or an abnormal hematocrit. All subjects gave written informed consent prior to participation. This study was approved by the Institutional Review Board of the Oriental Hospital of Daejeon University, Korea (approval no., djomc-24).

\section{Whole blood platelet aggregation assay}

Aggregation studies were performed with a 500VS Chrono-log aggregometer (Chrono-Log Co.), using the impedance method [19]. Intravenous blood for whole blood studies was drawn from healthy volunteers into Vacutainer tubes (Becton Dickinson, Franklin Park, NJ, USA) containing 3.8\% sodium citrate, quickly diluted with one volume of $0.9 \%$ saline solution, and used directly. Whole blood was incubated at $37^{\circ} \mathrm{C}$ in $450 \mu \mathrm{L}$ aliquots until the time of use. Samples were maintained at $37^{\circ} \mathrm{C}$ and stirred at $200 \times \mathrm{g}$ during aggregation testing. Following determination of the amount of agonist, such as collagen, AA, and ADP, necessary to cause a suitable impedance (W) full-scale deflection, varied sample doses in $50 \mu \mathrm{L}$ aliquots were added to $1 \mathrm{~mL}$ of diluted whole blood in a polystyrene cuvette (Chrono-Log Co.), $5 \mathrm{~min}$ prior to adding the agonist. The deflection observed at 8 min after adding the agonist was used for the calculation. Aggregation was quantified as the change in impedance. Several concentrations of EA-TCMG were used so that an $\mathrm{IC}_{50}$ value could be determined from a plot of concentration vs. percent inhibition.

\section{Thromboxane $B_{2}$ measurement}

Following the AA-induced aggregation assay, whole blood samples were centrifuged at 2,000 $\times \mathrm{g}$ for $15 \mathrm{~min}$ for the $\mathrm{TXB}_{2}$ immunoassay. Supernatant aliquots were immediately stored at $-20^{\circ} \mathrm{C}$ until assay. The concentration of $\mathrm{TXB}_{2}$, a stable metabolite of thromboxane $\mathrm{A}_{2}$ $\left(\mathrm{TXA}_{2}\right)$, was quantified using a TXB $\mathrm{TH}_{2}$ ELISA kit (R\&D Systems), according to the manufacturer's instructions. Absorbance was measured at $405 \mathrm{~nm}$ using a SpectaMAX 340 reader (Molecular Devices, Sunnyvale, CA, USA). $\mathrm{TXB}_{2}$ concentration was calculated from a standard curve.

\section{Animals and peripheral blood circulation distur- bance model}

Male ICR mice (Orient Bio Inc., Seongnam, Korea; weight, $25 \pm 3 \mathrm{~g}$ ) were maintained in cages under light- 
controlled conditions (12 h light/dark cycle) with ad libitum access to food and water. All experimental procedures were approved by the Animal Care and Use Committee of the Korea Institute of Oriental Medicine (approval no. 09-115). EA-TCMG or catechin was suspended in saline and administered orally to ICR mice at $500 \mathrm{mg} / \mathrm{kg}$ once daily for $3 \mathrm{~d}$ and once before initiating the experiment. All experiments were performed 1-h after the final administration. We previously developed the peripheral circulatory disturbance in vivo model [20]. Briefly, after induction with $2.5 \%$ isoflurane, animals were anesthetized with $1.5 \%$ isoflurane in a mixture of oxygen $(0.1 \mathrm{~L} / \mathrm{min})$ and nitrogen $(0.2 \mathrm{~L} / \mathrm{min})$. Peripheral blood flow in the mouse foot region was measured by laser Doppler flowmetry (LDF; BFL21, Transonic Instrument, Ithaca, NY, USA) and recorded using Chart4 for Windows software (ADI Instruments, New South Wales, Australia). Peripheral blood circulatory disturbance was induced in mice under cooling conditions using a temperature-controlled chamber (Chamlide IV; Live Cell Instruments, Seoul, Korea). The tissue perfusion unit value of stabilized peripheral blood flow was measured for $10 \mathrm{~min}$, and this value was set as the baseline using LDF. In addition, rectal temperature was measured at 5 min intervals. Peripheral blood flow was expressed as a peripheral blood flow ratio (peripheral blood flow value/ baseline value). Each mouse was observed for $100 \mathrm{~min}$ to determine inhibition or recovery from the lower blood flow caused by the peripheral circulatory disturbance.

\section{Statistical analysis}

All analyses were performed with the SPSS ver. 12.0 (SPSS Inc., Chicago, IL, USA). All results are presented as means \pm standard deviation. The $\mathrm{IC}_{50}$ values were calculated using the Regression Wizard from Sigma Plot. The inhibition of catechin or EA-TCMG on human platelet aggregation induced by collagen was calculated using the paired Student's t-test. Differences in $\mathrm{TXB}_{2}$ production were determined by one-way analysis of the variance (ANOVA) followed by Tukey's post-hoc test. The peripheral blood flow ratio differences between the two experimental groups were calculated by repeated measure ANOVA followed by Tukey's post-hoc test. Differences were statistically significant at $p$-values $<0.05$.

\section{RESULTS}

\section{Anti-platelet activity of ethyl acetate extract of tissue culture mountain ginseng in human whole blood}

EA-TCMG showed the greatest inhibition of human
Table 1. Inhibitory effect of the solvent fractions from a TCMG ethanol extract on human platelet aggregation induced by collagen

\begin{tabular}{lll}
\hline Samples & Solvent fractions $(500 \mu \mathrm{g} / \mathrm{mL})$ & \multicolumn{1}{c}{ Inhibition $(\%)$} \\
\hline Catechin & & $63.98 \pm 10.54$ \\
TCMG & $n$-hexane & $44.74 \pm 7.44$ \\
& Trichloromethane & $24.75 \pm 3.57$ \\
& Ethyl acetate & $93.06 \pm 1.84(p=0.004)^{1)}$ \\
& $n$-butanol & $17.42 \pm 1.07$ \\
& $\mathrm{H}_{2} \mathrm{O}$ & $22.25 \pm 3.57$ \\
\hline
\end{tabular}

Data are mean \pm SD.

TCMG, tissue-cultured mountain ginseng.

${ }^{1)} p$-value in comparison to catechin as a reference by the paired Student's $t$-test.

whole blood platelet aggregation in a preliminary screening of solvent fractions. Using the impedance method of aggregometry, we tested the in vitro effect of solvent fractions from the TCMG ethanol extract on platelet aggregation induced by collagen in whole human blood. Table 1 lists the inhibitory effects of the solvent fractions on human platelet aggregation. Inhibition percentages for EA-TCMG were $93.06 \pm 1.84 \%$, whereas values for $n$-hexane, trichloromethane, $n$-butanol and $\mathrm{H}_{2} \mathrm{O}$ extracts, all at a $500 \mu \mathrm{g} / \mathrm{mL}$, were $44.74 \pm 7.44 \%, 24.75 \pm 3.57 \%$, $17.42 \pm 1.07 \%$, and $22.25 \pm 3.57 \%$, respectively. Among them, EA-TCMG $(93.06 \pm 1.84 \%, p=0.004)$ was selected as an effective fraction in comparison with catechin as a control agent $(63.98 \pm 10.54 \%)$. Fig. 1 shows the inhibitory activity of EA-TCMG on human platelet aggregation induced by agonists such as AA, ADP, and collagen in a dose-dependent manner and the pattern for the typical curves. Table 2 shows the $\mathrm{IC}_{50}$ values for EA-TCMG calculated from the concentration-inhibition curves as 271, 180 , and $147 \mu \mathrm{g} / \mathrm{mL}$ for collagen, ADP, and AA-induced platelet aggregation, respectively.

\section{The inhibitory effects on thromboxane $B_{2}$ produc- tion}

$\mathrm{TXA}_{2}$ is rapidly hydrolyzed to $\mathrm{TXB}_{2}$, a stable plasma product of the cyclooxygenase pathway. $\mathrm{TXA}_{2}$ is a potent stimulator of platelet aggregation. Therefore, we examined whether EA-TCMG blocked the production of $\mathrm{TXB}_{2}$ under AA exposure. The $\mathrm{TXA}_{2}$ (determined as $\mathrm{TXB}_{2}$ ) production percentages in the EA-TCMG and catechin treatments at $500 \mu \mathrm{g} / \mathrm{mL}$ were $68.64 \pm 8.57$ and $56.42 \pm 9.44 \%$ when compared to the control group (saline+AA), respectively. As shown in Fig. 2, EA-TCMG mildly reduced $\mathrm{TXB}_{2}$ production but significantly inhibited $\mathrm{TXB}_{2}$ production at $500 \mu \mathrm{g} / \mathrm{mL}$. 
Catechin
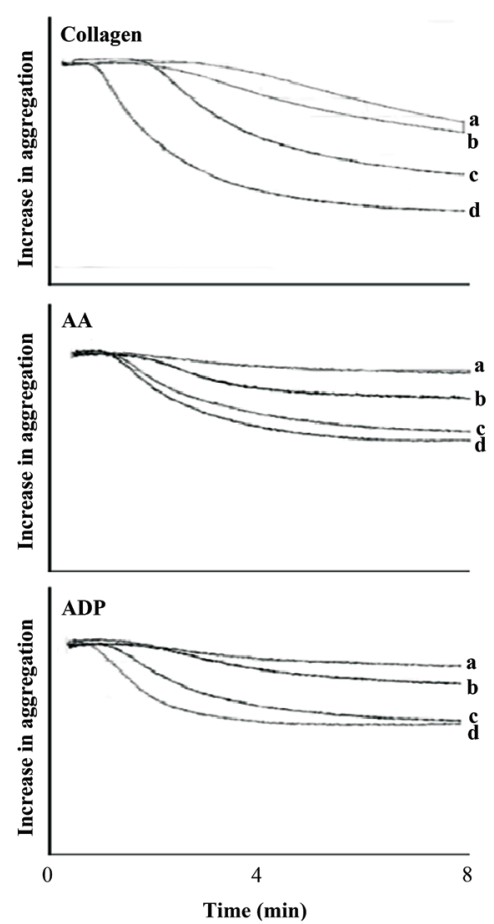

A

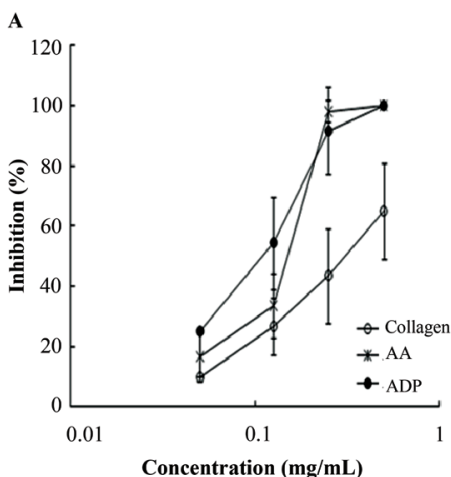

EA-TCMG
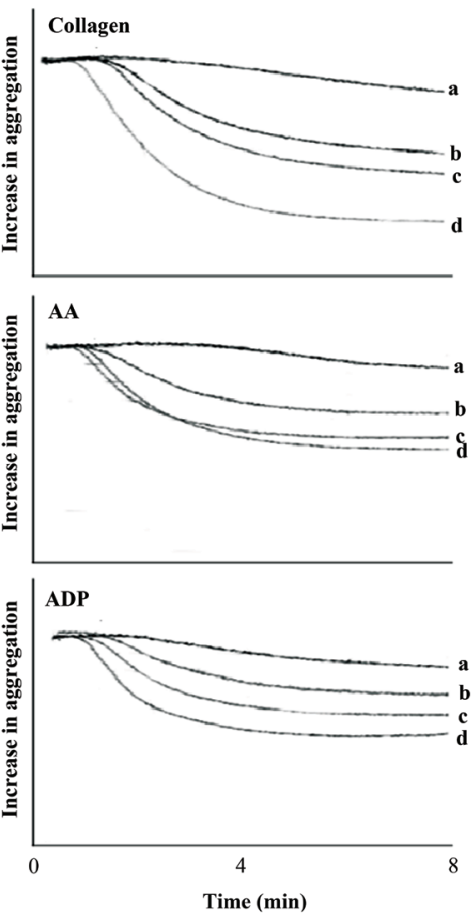

B

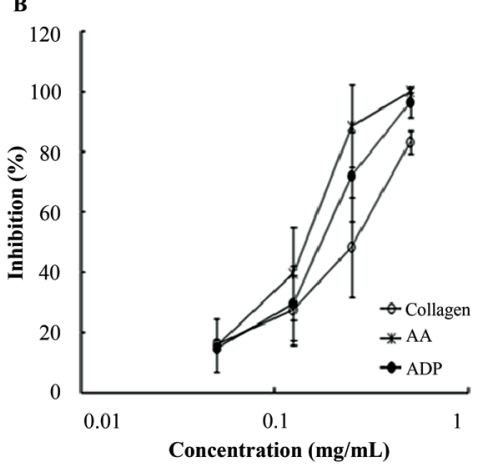

Fig. 1. Representative plots showing the pattern for typical curves of collagen-, arachidonic acid (AA)-, and adenosine-5'-diphosphate (ADP)induced platelet aggregation obtained with dose-dependent samples of the ethyl acetate extract of tissue culture mountain ginseng (EA-TCMG) extract or catechin. a: $500 \mu \mathrm{g} / \mathrm{mL}$, b: $250 \mu \mathrm{g} / \mathrm{mL}$, c: $125 \mu \mathrm{g} / \mathrm{mL}$, d: saline. Aggregation in the presence of the EA-TCMG extracts or catechin is expressed as a percentage of that in the absence of the extract. Results are mean $\pm S D$. (A) Inhibitory effects of catechin as a control agent. (B) Inhibitory effects of EA-TCMG extract.

Table 2. $\mathrm{IC}_{50}$ values of EA-TCMG on human platelet aggregation induced by various agonists

\begin{tabular}{lcc}
\hline \multirow{2}{*}{ Agonist } & \multicolumn{2}{c}{$\mathrm{IC}_{50}$ value $^{1)}(\mu \mathrm{g} / \mathrm{mL})$} \\
\cline { 2 - 3 } & Catechin & EA-TCMG \\
\hline Collagen $(2 \mu \mathrm{g} / \mathrm{mL})$ & 342 & 271 \\
AA $(0.5 \mathrm{mM})$ & 143 & 147 \\
ADP $(15 \mu \mathrm{M})$ & 120 & 180 \\
\hline
\end{tabular}

$\mathrm{IC}_{50}, 50 \%$ inhibitory concentration; EA-TCMG, ethyl acetate extract of tissue culture mountain ginseng; AA, arachidonic acid; ADP, adenosine-5'-diphosphate.

${ }^{1)}$ Calculated from a dose-dependent curve using the Regression Wizard in Sigma Plot.

\section{EA-TCMG alleviates the peripheral blood circula- tion disturbance in mice}

A peripheral blood circulatory disturbance was induced in mice under cooling conditions using a temperature-controlled chamber [20]. To examine the effects of EA-TCMG on improving peripheral blood circulation in the in vivo model, peripheral blood flow was compared between the experimental group $(n=8$, EA-TCMG oral administration), the control group ( $n=8$, saline oral administration), and the positive drug group ( $n=8$, catechin oral administration). Rectal temperatures were similar 


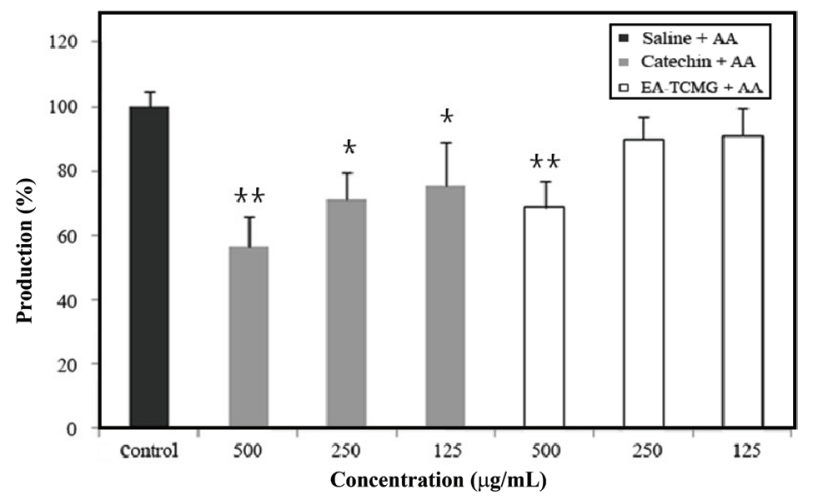

Fig. 2. Effects of an ethyl acetate extract of tissue culture mountain ginseng (EA-TCMG) on the production of thromboxane $B_{2}\left(T X B_{2}\right)$ induced by arachidonic acid (AA). Whole blood was preincubated with the EA-TCMG extract for $5 \mathrm{~min}$ and then stimulated with AA $(0.5$ $\mathrm{mM}$ ). $\mathrm{TXB}_{2}$ was investigated as described in Materials and Methods. The amount produced was measured using a $\mathrm{TXB}_{2}$ EIA kit. Values are given as mean $\pm \mathrm{SD}$. ${ }^{*} p<0.05,{ }^{* *} p<0.01$ represents a significant difference between the experimental and control values. among the EA-TCMG, catechin, and saline experimental groups. Peripheral blood flow was severely reduced after cooling in all mice. After $30 \mathrm{~min}$, peripheral blood flow ratios in the EA-TCMG and saline groups decreased to $0.77 \pm 0.22$ and $0.63 \pm 0.20$, respectively. Note that the decrease in the saline group was induced by cooling. After $60 \mathrm{~min}$, peripheral blood flow ratios in the EA-TCMG group and the saline group were $0.21 \pm 0.07$ and $0.19 \pm 0.07$, respectively. During $60 \mathrm{~min}$ after measurement, the improvement in peripheral blood flow was mild in the EATCMG (500 mg/kg, $p=0.041)$ group compared with that in saline group but significant in the catechin group (500 $\mathrm{mg} / \mathrm{kg}, p=0.001$ ) (Fig. 3). These results indicate that the peripheral blood flow ratio decreased in both groups; however, the decrease in the blood flow ratio was lower in EA-TCMG group than that in the saline group. These findings indicate that the EA-TCMG treatment was effective for improving peripheral blood flow.
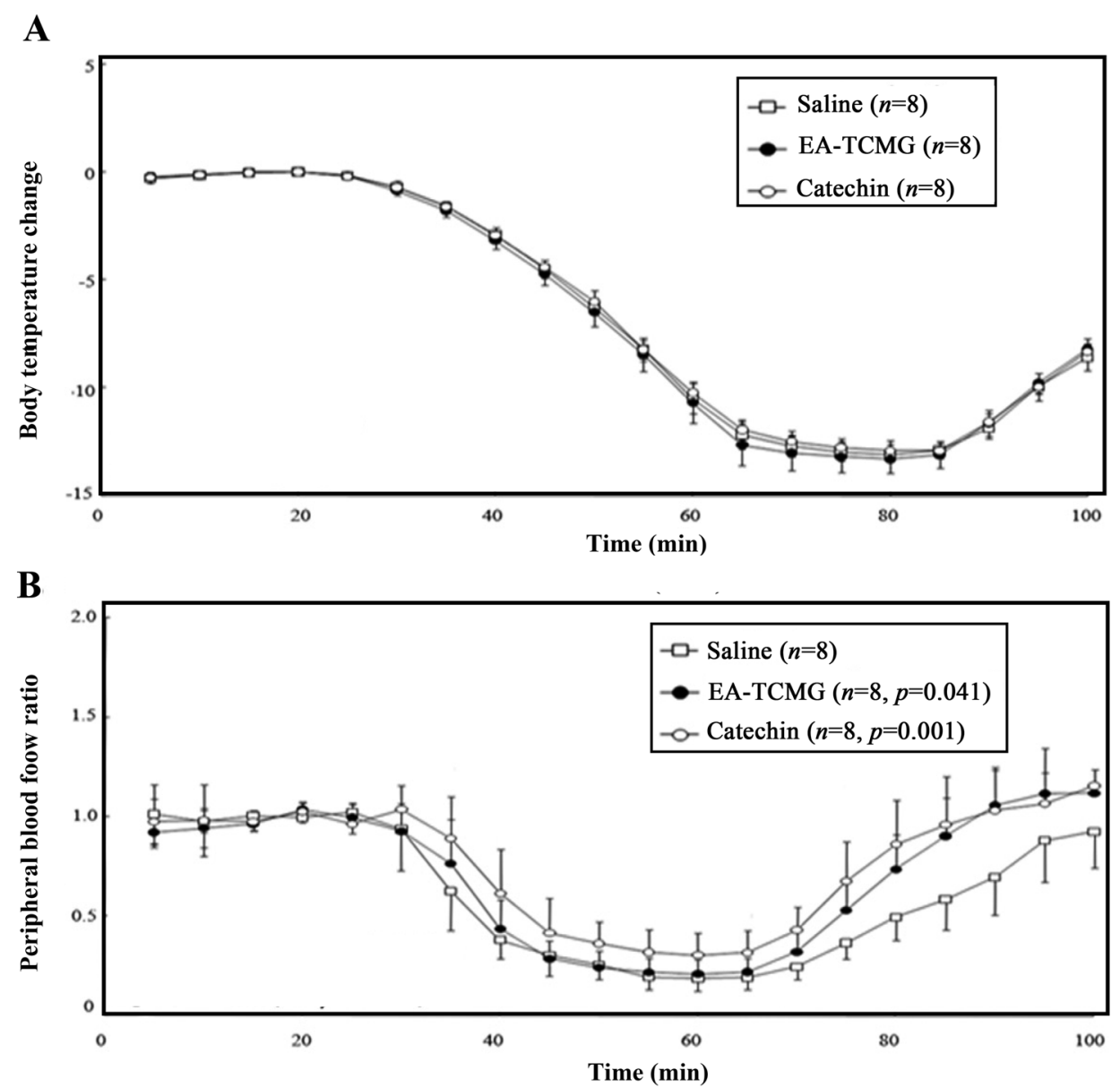

Fig. 3. Effects of an ethyl acetate extract of tissue culture mountain ginseng (EA-TCMG) on peripheral blood circulation disturbance in mice. Change in average peripheral blood flow ratio and temperature distribution during temperature-controlled chamber cooling and heating phases after oral administration of EA-TCMG $(500 \mathrm{mg} / \mathrm{kg})$. (A) Time course of change in rectal temperature. (B) Time course of change in peripheral blood flow ratio (peripheral blood flow value/baseline value). Data are mean \pm SD. A $p$-value in comparison to vehicle control (saline) by repeatedmeasures analysis of variance with a Tukey's post-hoc means comparison. 


\section{DISCUSSION}

We have reported that TCMG adventitious root ethanol extract has anti-platelet activity in human whole blood, compared with ginseng commercial products [15]. We then selected the TCMG extract as a candidate for further anti-platelet activity study. In this study, we investigated the in vitro effect of EA-TCMG on platelet aggregation and then evaluated the in vivo effects on a peripheral circulatory disturbance in a mouse model. Our data on antiplatelet activity in human whole blood show that EATCMG could be protective against platelet aggregation (Fig. 1), and that it reduced $\mathrm{TXB}_{2}$ production induced by various agonists (Fig. 2). In particular, EA-TCMG suppressed release of AA from platelet membrane phospholipids and significantly reduced formation of $\mathrm{TXB}_{2}$, as a biomarker of platelet activation. The inhibitory effect of EA-TCMG on AA release and $\mathrm{TXB}_{2}$ formation may contribute to reduced platelet aggregation. Irreversible platelet aggregation and vasoconstriction caused by $\mathrm{TXA}_{2}$ formation has been reported [21]. Experimental animal models, such as the acute pulmonary thromboembolism [22], the arteriovenous shunt thrombosis [23] and the arterial thrombosis models have been developed to evaluate anti-platelet or anti-thrombotic agents [24]. However, peripheral circulatory disturbance in an in vivo model, which occurs as a result of changes in blood flow, has not been established. Several studies have reported on factors causing sensitive changes in blood flow [25,26]. Of the factors that affect blood flow, changes in external temperature trigger a change in skin temperature, which, in turn, causes changes in blood flow, because long-term exposure of the blood vessels to a cold-temperature environment and the resulting vasoconstriction cause abnormalities in blood flow, thereby inducing platelet aggregation [27]. Similarly, exposure of the whole body to a low temperature results in decreased blood flow [26]. Based on these studies, we previously developed an in vivo model of peripheral circulatory disturbance to confirm the efficiency of aspirin [20]. In this study, our results indicate that oral administration of EA-TCMG was effective for improving peripheral blood flow using the model of peripheral disturbance circulation. These results confirmed the efficacy, including improvement in peripheral blood circulation. In conclusion, EA-TCMG, as a natural product derived from tissue culture techniques, has a potential effect on blood circulation, as determined by an in vitro whole blood aggregation assay and an in vivo model of peripheral blood circulatory disturbance. These results suggest that EA-TCMG has potent anti-platelet activity and that it improves peripheral blood circulation. Further elucidation of the active compounds in EA-TCMG could lead to the development of potent anti-platelet agents for preventing various vascular diseases; however mechanistic studies are necessary to define the effectiveness of EA-TCMG.

\section{ACKNOWLEDGEMENTS}

This study was supported by the development project of agricultural technology of Ministry for Food, Agriculture, Forestry and Fisheries. This study was also supported, in part, by a grant (kiom-2010-2) from the InterInstitutional Collaboration Research Program provided by the Korea Research Council of Fundamental Science \& Technology, Korea.

\section{REFERENCES}

1. Xiang YZ, Kang LY, Gao XM, Shang HC, Zhang JH, Zhang BL. Strategies for antiplatelet targets and agents. Thromb Res 2008;123:35-49.

2. Hubbard GP, Stevens JM, Cicmil M, Sage T, Jordan PA, Williams CM, Lovegrove JA, Gibbins JM. Quercetin inhibits collagen-stimulated platelet activation through inhibition of multiple components of the glycoprotein VI signaling pathway. J Thromb Haemost 2003;1:1079-1088.

3. Nam KY. The comparative understanding between red ginseng and white ginsengs, processed ginsengs (Panax ginseng C. A. Meyer). J Ginseng Res 2005;29:1-18.

4. Seog HM, Jung CH, Kim YS, Park HS. Phenolic acids and antioxidant activities of wild ginseng (Panax ginseng C. A. Meyer) leaves. Food Sci Biotechnol 2005;14:371374.

5. Kim YS, Hahn EJ, Murthy HN, Paek KY. Adventitious root growth and ginsenoside accumulation in Panax ginseng cultures as affected by methyl jasmonate. Biotechnol Lett 2004;26:1619-1622.

6. Paek KY, Murthy HN, Hahn EJ, Zhong JJ. Large scale culture of ginseng adventitious roots for production of ginsenosides. Adv Biochem Eng Biotechnol 2009;113:151-176

7. Sivakumar G. Bioreactor technology: a novel industrial tool for high-tech production of bioactive molecules and biopharmaceuticals from plant roots. Biotechnol J 2006;1:1419-1427.

8. Park JS, Hwang SY, Lee WS, Yu KW, Paek KY, Hawng BY, Han K. The therapeutic effect of tissue cultured root of wild Panax ginseng C.A. Mayer on spermatogenetic disorder. Arch Pharm Res 2006;29:800-807. 
9. Woo SH, Eom MS, Shin KH, Han KH, Kim WJ. The neutralizing effect of Panax ginseng for toxicities in the survival, sperm quality, pregnancy and F1 generation of guinea pigs exposed to 2,3,7,8-tetrachlorodibenzo-pdioxin. Korean J Urol 2002;43:161-168.

10. Kim HS, Woo SH, Jo S, Hahn EJ, Youn NY, Lee HL. Double-blind, placebo-controlled, multi-center study for therapeutic effects of mountain Panax ginseng C.A. Meyer extract in men with erectile dysfunction: a preliminary report. Korean J Androl 2006;24:84-88.

11. Lee EJ, Zhao HL, Li DW, Jeong CS, Kim JH, Kim YS, Effect of the $\mathrm{MeOH}$ extract of adventious root culture of Panax ginseng on hyperlipidemic rat induced by high fatrich diet. Korean J Pharmacognosy 2003;34:179-184.

12. Ali MB, Hahn EJ, Paek KY. Protective role of Panax ginseng extract on lipid peroxidation and antioxidant status in polyethylene glycol induced Spathiphyllum leaves. Biochem Eng J 2006;32:143-148.

13. Lim HK, Kim YW, Lee DH, Kim SM, Cho MJ. The antifibrotic and antioxidant activities of hot water extract of adventitious root culture of Panax ginseng (ARCP). J Appl Biol Chem 2007;50:78-84.

14. Oh CH, Kang PS, Kim JW, Kwon J, Oh SH. Water extracts of cultured mountain ginseng stimulate immune cells and inhibit cancer cell proliferation. Food Sci Biotechnol 2006;15:369-373.

15. Jeon WK, Yo BK, Kim YE, Park SO, Hahn EJ, Paek KY, Ko BS. Anti-platelet activity of tissue-cultured mountain ginseng adventitious roots in human whole blood. Food Sci Biotechnol 2008;17:1197-1202.

16. Ni H, Freedman J. Platelets in hemostasis and thrombosis: role of integrins and their ligands. Transfus Apher Sci 2003:28:257-264.

17. Jin JL, Lee YY, Heo JE, Lee SH, Kim JM, Yun-Choi HS. Anti-platelet pentacyclic triterpenoids from leaves of Campsis grandiflora. Arch Pharm Res 2004;27:376-380.

18. Lee YY. Biological effects of components isolated from Angelica genuflexa [dissertation]. Seoul: Seoul National
University, 2004.

19. Torres Duarte AP, Dong QS, Young J, Abi-Younes S, Myers AK. Inhibition of platelet aggregation in whole blood by alcohol. Thromb Res 1995;78:107-115.

20. Yoo BK, Ko BS, Ma JY, Jeon WK. Efficacy of aspirin on improvement of peripheral blood circulation in mice. $\mathrm{J}$ Korean Soc Appl Biol Chem 2009;52:22-27.

21. Huang J, Wang S, Luo X, Xie Y, Shi X. Cinnamaldehyde reduction of platelet aggregation and thrombosis in rodents. Thromb Res 2007;119:337-342.

22. Hsiao G, Shen MY, Lin KH, Chou CY, Tzu NH, Lin $\mathrm{CH}$, Chou DS, Chen TF, Sheu JR. Inhibitory activity of kinetin on free radical formation of activated platelets in vitro and on thrombus formation in vivo. Eur J Pharmacol 2003;465:281-287.

23. Umar A, Guerin V, Renard M, Boisseau M, Garreau C, Begaud B, Molimard M, Moore N. Effects of armagnac extracts on human platelet function in vitro and on rat arteriovenous shunt thrombosis in vivo. Thromb Res 2003;110:135-140.

24. Konstantinides S, Schafer K, Thinnes T, Loskutoff DJ. Plasminogen activator inhibitor-1 and its cofactor vitronectin stabilize arterial thrombi after vascular injury in mice. Circulation 2001;103:576-583.

25. Chida Y, Sudo N, Kubo C. Psychological stress impairs hepatic blood flow via central CRF receptors in mice. Life Sci 2005;76:1707-1712.

26. Sawasaki N, Iwase S, Mano T. Effect of skin sympathetic response to local or systemic cold exposure on thermoregulatory functions in humans. Auton Neurosci 2001;87:274-281.

27. Rusyniak DE, Ootsuka Y, Blessing WW. When administered to rats in a cold environment, 3,4-methylenedioxymethamphetamine reduces brown adipose tissue thermogenesis and increases tail blood flow: effects of pretreatment with 5-HT1A and dopamine D2 antagonists. Neuroscience 2008;154:1619-1626. 